\title{
Spatio-temporal Differentiation in the Incidence of Influenza and Its Relationship with Air Pollution in China from 2004 to 2017
}

\author{
SONG Yang ${ }^{1,2}$, ZHANG Yu ${ }^{1}$, WANG Tingting ${ }^{1}$, QIAN Sitong ${ }^{1}$, WANG Shijun ${ }^{1,2}$ \\ (1. School of Geographical Sciences, Northeast Normal University, Changchun 130024, China; 2. Key Laboratory of Geographical \\ Processes and Ecological Security in Changbai Mountains, Ministry of Education, Changchun 130024, China)
}

\begin{abstract}
The Healthy China Initiative is a major health strategy being pursued by the country. To prevent and control different types of diseases as well as their complex variants, research on the spatio-temporal differentiation among and mechanisms of influence of epidemic diseases is growing worldwide. This study analyzed monthly data on the incidence of influenza by using different methods, including Moran's I, the hotspot analysis model, concentration analysis, and correlation analysis, to determine the characteristics of spatiotemporal differentiation in the incidence of influenza across prefecture-level cities in China from 2004 to 2017 , and to examine its relationship with air pollution. According to the results, the overall incidence of influenza in China exhibited a trend of increase from 2004 to 2017, with small peaks in 2009 and 2014. More cases of influenza were recorded in the first and fourth quarters of each year. Regions with higher incidences of influenza were concentrated in northwestern and northern China, and in the coastal areas of southeastern China. Over time, the distribution of regions with a higher incidence of influenza has shifted from the west to the east of the country. A significant relationship was observed between the incidence of influenza and factors related to air pollution. The contents of five air pollutants $\left(\mathrm{PM}_{2.5}, \mathrm{PM}_{10}, \mathrm{SO}_{2}, \mathrm{NO}_{2}\right.$, and $\left.\mathrm{CO}\right)$ were significantly positively correlated with the incidence of influenza, with a decreasing order of contribution to it of $\mathrm{SO}_{2}>\mathrm{CO}>\mathrm{NO}_{2}>\mathrm{PM}_{2.5}>\mathrm{PM}_{10}$. The content of $\mathrm{O}_{3}$ in the air was negatively correlated with the incidence of influenza. The influence of air pollution-related factors on the incidence of influenza in different regions and seasons showed minor differences. The large-scale empirical results provided here can supply a scientific basis for governmental disease control authorities to formulate strategies for regional prevention and control.
\end{abstract}

Keywords: incidence of influenza; spatio-temporal differentiation; air pollution; China

Citation: SONG Yang, ZHANG Yu, WANG Tingting, QIAN Sitong, WANG Shijun, 2021. Spatio-temporal Differentiation in the Incidence of Influenza and Its Relationship with Air Pollution in China from 2004 to 2017. Chinese Geographical Science, 31(5): 815-828. https://doi.org/10.1007/s11769-021-1228-2

\section{Introduction}

The rapid pace of industrialization and urbanization has led to drastic changes in the modes of production, people's lifestyle, and the environment that have profound impacts on human health and the spectrum of dis- eases threatening it. This state of affairs has increased the instability, complexity, and vulnerability of the national health system in China (Yang et al., 2018). Diseases that can turn into pandemics are increasing in variety. Influenza (or the flu) is an acute respiratory infectious disease caused by the influenza virus that spreads

Received date: 2021-01-18; accepted date: 2021-03-25

Foundation item: Under the auspices of Key Program of National Natural Science Foundation of China (No. 41630749), Program of the National Social Science Fund of China (No. 17BJL051), Fundamental Research Funds for the Central Universities (No. 1709103, 2412020FZ001)

Corresponding author: WANG Shijun. E-mail: wangsj@nenu.edu.cn

(C) Science Press, Northeast Institute of Geography and Agroecology, CAS and Springer-Verlag GmbH Germany, part of Springer Nature 2021 
through the air (National Health Commission of the People's Republic of China, 2020). It is highly infectious, transmits quickly, and can easily lead to outbreaks and even pandemics (Hilleman, 2002). Influenza outbreaks harm human health and impose a heavy economic burden, and thus are a serious global public health problem (Jamieson et al., 2009; Matus et al., 2012; Bhuiyan et al., 2014; Putri et al., 2018). According to the World Health Organization (WHO), seasonal influenza epidemics cause 650000 deaths across the world each year, equivalent to a person dying from influenza every 48 seconds (WHO, 2018). The incidence rate and mortality rate of influenza are relatively low, compared with other infectious diseases with the highest annual incidence rate: hand foot mouth disease, hepatitis $\mathrm{B}$, tuberculosis and other infectious diarrhea and the highest annual mortality rate: tuberculosis, rabies, AIDS (Acquired Immune Deficiency Syndrome) and viral hepatitis from 2002 to 2017, but the trend has been increasing in recent years ( $\mathrm{Li}$ and $\mathrm{Zhu}, 2019$ ). The Healthy China Initiative is a significant health strategy being pursued by the country (The Central People's Government of the People's Republic of China, 2019). It involves strengthening the prevention and control of severe infectious diseases, improving the monitoring of and early warning mechanism for infectious diseases, and improving urban air quality nationwide to ensure that it accords with the relevant standards. In light of these goals, regional differences in the incidence of influenza, the effect of poisonous and harmful pollutants on human health, the specific relationship between influenza and changes in air pollution-related factors, and targeted measures for regional environmental governance to mitigate the effects of infectious diseases are issues that need to be systematically studied.

Public health has long been a core area of research in international geography and environmental science (Yang et al., 2010). The role of geography in public health has become prominent in recent years by highlighting its spatial perspective, and revealing that the spatio-temporal distribution of diseases exhibits significant regional characteristics. Research in the area has focused on laws of the spatio-temporal distribution of diseases and the relevant health conditions (Artois et al., 2018; Guinat et al., 2018; Gong et al., 2020), models for the spatial transmission of infectious diseases (Wang et al., 2020), balancing and regulating the relationships among the environment, development, and health (Zhang et al., 2007), medico-geographical and health-related risk assessments (Shkurinskii and Chibilev, 2012; Murari et al., 2020), regional allocation of healthcare systems and facilities (Holley, 1998; Trujillo and Plough, 2016), and the relation between the urban microenvironment and health (Rosenberg, 2017; Qi and Zhou, 2018; Yang et al., 2019).

The major methods used to study epidemic diseases include descriptive epidemiology (Zhang, 2013), spatial agglomeration analysis ( $\mathrm{Lu}$ et al., 2010; Lin et al., 2020), and landscape genetics (Biek and Real, 2010). The transmission of diseases is influenced by natural factors, such as air pollution and meteorological conditions (Dangi et al., 2014; Skog et al., 2014; Vittecoq et al., 2015; Lelieveld et al., 2015; Sun et al., 2018), and socio-economic factors such as income, consumption, age, and social security (Wu et al., 2009; VazquezProkopec et al., 2010; Mahabir et al., 2012; Li et al., 2013; Wen et al., 2015). Studies have shown that long and short-term exposure to $\mathrm{PM}_{2.5}, \mathrm{NO}_{2}, \mathrm{SO}_{2}$, and $\mathrm{PM}_{10}$ is closely associated with cardiovascular and respiratory diseases, and may be a significant contributor to cancer (Utell and Frampton, 2000; Lonati et al., 2011; To et al., 2015; Hayes et al., 2020).

Current research on influenza has focused on its epidemiology. The agglomeration of influenza (Chen et al., 2011; Lee and Wong, 2011; Yang et al., 2011) and the epidemiological characteristics (Wang et al., 2017) of its occurrence differ significantly at different spatial scales. Scholars have focused on analyzing the scale of diseases in regions of different sizes. Systematic long-term and small-scale epidemiological data for China are lacking, and the analysis of the impact of environmental factors, such as air pollution, on diseases in the country has been limited. Medical workers have used statistical analysis to describe the epidemiological characteristics and social burden of influenza and other diseases. Analytical research on spatial differences among the incidences of influenza and natural factors influencing them is still in its infancy. To address this gap in research, this study collected data on the incidence of influenza and air pollution in China. We used Moran's $I$, the hotspot analysis model, concentration analysis, and Spearman's rank correlation analysis to study the characteristics of the spatio-temporal distribution of and factors influencing the spread of influenza. This study has three aims: 
1) to provide large-scale empirical data on influenza that can be used for health geography, 2) to determine the mechanism of influence of air pollution-related factors on the incidence of influenza from multiple perspectives and identify its spatio-temporal evolution, and 3) to provide insights that can be used by disease control authorities to formulate regional prevention and control strategies, and provide theoretical support for the implementation of the Healthy China Initiative.

\section{Materials and Methods}

\subsection{Data and processing}

We used data from 31 provinces, municipalities and autonomous regions of China (excluding Hong Kong, Macau, and Taiwan of China) in this study, with the prefecture-level city as the basic unit of the scale. The research period ranged from 2004 to 2017 . The collected information included data on the incidence of influenza and air quality, and maps. Monthly data on the incidence of influenza in prefectural cities were used. All reported data were used as samples to analyze the spatial and temporal characteristics of the incidence of influenza. The number of cities for which annual data on the incidence of influenza were available increased from 257 in 2004 to 367 in 2017. The data were drawn from Data Center of China Public Health Science, part of China Center for Disease Control and Prevention (http://www.phsciencedata.cn/share/). Given the small number of deaths recorded, data on only the number of cases of influenza were used, where incidence $=$ the number of cases $\times 100000$ people. Statistical data for some regions for a number of years were missing, because of which the data on influenza were not spatially continuous. The missing data were set as blank, without interpolation. The layers of data for administrative maps of prefecture-level cities were obtained from the Geographic Information Bureau of the State Bureau of Surveying and Mapping. Pollutant indices for air quality were obtained from the real-time air quality monitoring data published by the China National Environmental Monitoring Center (http://www.cnemc.cn/), and included data on fine particulate matter $\left(\mathrm{PM}_{2.5}\right)$, inhalable particulate matter $\left(\mathrm{PM}_{10}\right)$, sulfur dioxide $\left(\mathrm{SO}_{2}\right)$, nitrogen dioxide $\left(\mathrm{NO}_{2}\right)$, ozone $\left(\mathrm{O}_{3}\right)$, and carbon monoxide (CO). A total of 190 cities were monitored daily for air quality in 2014 and 366 in 2015-2017, and the monthly mean value for each was calculated from the daily data. When analyzing the relationship between air pollution and the incidence of influenza, cities for which data on air quality and the incidence of influenza were available were selected as samples. The numbers of samples (cities) used for 2014 to 2017 were 160, 327, 328, and 330, respectively. The differences in the numbers of samples between years affected statistical efficiency but did not compromise the statistical inferences made because the total coverage was large enough.

\subsection{Methodology}

\subsubsection{Moran's Global I}

Moran's global $I$ statistic is the most commonly measure used to analyze the overall spatial correlation and degrees of difference in a given study area (Haining, 2003). We used it to investigate the overall spatial distribution of the incidence of influenza across China.

\subsubsection{Getis-Ord $G_{i}{ }^{*}$}

The local Getis-Ord $G_{i}{ }^{*}$ hotspot detection is a typical statistical method of local spatial autocorrelation that can be used to identify spatial variation (Getis and Ord, 1992). It can accurately reflect the distribution of hotspots for influenza in a given area (Wang et al., 2014).

\subsubsection{Concentration analysis}

Concentration analysis involves the use of daytime and seasonal data to analyze the degree of concentration of influenza in a given community over the course of a year (Liu, 1985). It is calculated as follows:

$R_{x}=\frac{1}{2}\left(r_{2}+r_{6}-r_{8}-r_{12}\right)+\frac{\sqrt{3}}{2}\left(r_{3}+r_{5}-r_{9}-r_{11}\right)+\left(r_{4}-r_{10}\right)$

$R_{y}=\frac{1}{2}\left(r_{3}-r_{5}-r_{9}+r_{11}\right)+\frac{\sqrt{3}}{2}\left(r_{2}-r_{6}-r_{8}+r_{12}\right)+\left(r_{1}-r_{7}\right)$

$M=\sqrt{\left(R_{x}\right)^{2}+\left(R_{y}\right)^{2}}$

where $r_{i}$ denotes the incidence of influenza in a month as a percentage of its annual incidence, $i$ denotes the month, $R$ denotes dispersion, $R_{x}$ and $R_{y}$ denote dispersion along the $x$-axis and the $y$-axis, respectively, and $M$ denotes concentration. When $M=1$, this indicates that the incidence of influenza is concentrated in a certain month, a value in the interval $[0.9,1)$ indicates strict seasonality, that in $[0.7,0.9]$ indicates very strong sea- 
sonality, values in the range $[0.5,0.7)$ indicate relatively strong seasonality, $[0.3,0.5)$ indicates a certain seasonality, and $[0,0.3)$ indicates insignificant seasonality. When the concentration is zero, this indicates a uniform distribution of the incidence of influenza over 12 months (Li et al., 2016).

\subsubsection{Spearman's rank correlation coefficient}

Air quality data are not continuously equidistant, and their forms of correlation and patterns of distribution are unknown. If Pearson's rank correlation coefficient is directly used for analysis, the results may be inaccurate. To improve the reliability of correlation analysis, Spearman's rank correlation analysis, a non-parametric correlation coefficient, was used in this study to analyze the degree of correlation between the incidence of influenza and factors influencing air quality (Spearman, 1904). This coefficient has no requirements regarding the selection of raw data, the form of the correlation, and the pattern of distribution. It is typically used to measure the strength of a monotonic relationship or rank correlation between variables. Its universality and robustness are better than those of Pearson's product moment correlation coefficient with the parametric property (Spearman, 1904).

\section{Results}

\subsection{Characteristics of temporal variation in the in- cidence of influenza}

\subsubsection{Annual variation}

Fig. 1 shows an overall trend of increase in the incidence of influenza in 31 provinces, municipalities and autonomous regions of China (excluding Hong Kong, Macao, and Taiwan of China) from 2004 to 2017. In 2006, it declined slightly and reached its minimum value in 2008. In 2009, it increased significantly due to the outbreak of influenza A (H1N1), then decreased, and then increased again. It fluctuated until reaching its maximum value in 2017. This was in line with the global influenza epidemic in 2017 (Du et al., 2018). The annual trend of variations in the occurrence of influenza

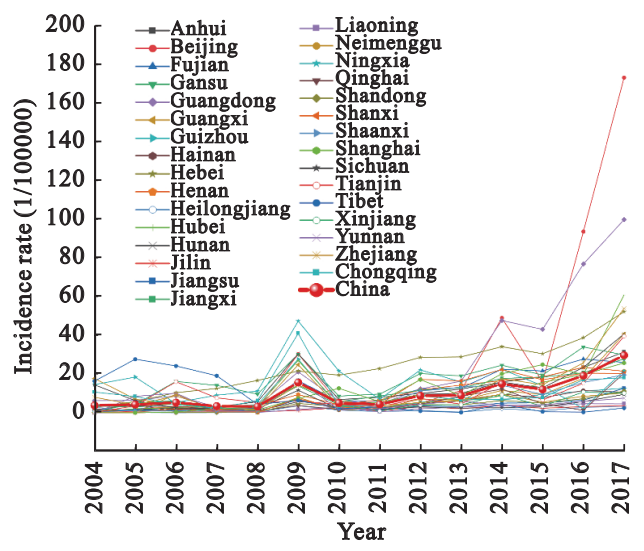

Fig. 1 The incidence of influenza in different provinces, municipalities and autonomous regions of China (excluding Hong Kong, Macau and Taiwan) during 2004-2017

were the same in most provinces and cities in China, and only a few provinces exhibited some differences. Most provinces, municipalities and autonomou regions registered a trend of increase, with fluctuations, and a peak in 2009, such as Ningxia and Chongqing. Three provinces in the northeastern China (Heilongjiang, Jilin and Liaoning) had slightly different trends, generally showing smaller fluctuations and lower incidence of influenza.

The characteristics of occurrence of influenza in some provinces, municipalities and autonomous regions differed substantially from the rest. For example, Beijing showed the largest annual differences, and had a much higher incidence of influenza than the other provinces, municipality and autonomous in China in 2014 and 2017. From 2014 to 2017, the incidence in Guangdong was two to three times higher than the national average. These substantial differences were based not only on the outbreak of influenza in 2017, but also on the number of data samples obtained from this region. That is, Guangdong might have recorded an increase in cases of influenza due to more testing and fewer missed diagnoses than the other regions.

\subsubsection{Seasonal variation}

Table 1 shows that the concentration of the incidence of influenza in China was mostly in the range of $[0.3,0.5)$, only slightly higher than that of 0.5 in 2009 . This indic-

Table 1 Degree of concentration analysis of influenza incidence in China (excluding Hong Kong, Macau and Taiwan) during 2004-2017

\begin{tabular}{|c|c|c|c|c|c|c|c|c|c|c|c|c|c|c|}
\hline Year & 2004 & 2005 & 2006 & 2007 & 2008 & 2009 & 2010 & 2011 & 2012 & 2013 & 2014 & 2015 & 2016 & 2017 \\
\hline The degree of concentration & 0.267 & 0.228 & 0.564 & 0.377 & 0.250 & 0.506 & 0.328 & 0.256 & 0.290 & 0.334 & 0.331 & 0.116 & 0.447 & 0.332 \\
\hline
\end{tabular}


ates that influenza in China generally exhibited seasonal characteristics, with the strongest seasonality registered in 2009 and the weakest in 2015.

A comparison of the monthly data (Fig. 2) showed that the incidence of influenza was mainly concentrated in the first and fourth quarters of each year, i.e., a period of higher incidence and susceptibility lasted from November of each given year to March of the following year. This might be because the transmission of the influenza virus by aerosol depends on the temperature and relative humidity. The low temperature and relative humidity in winter are more conducive to the transmission of influenza than in summer (Ma et al., 2019). In 2009 and 2016-2017, irregular fluctuations occurred, with a higher incidence of influenza than during the stable period. Its incidence in December 2017 was significantly higher than in the corresponding month in other years, which was related to the increase of influenza virus types in winter in 2017 (World Health Organization, 2017).

\subsection{Spatial agglomeration and variations in incid- ence of influenza}

\subsubsection{Overall spatial pattern}

Fig. 3 shows a clear differentiation in spatial patterns of the incidence of influenza in China, and substantial changes occurred during the study period. Regions with a higher incidence of influenza tended to shift from the west to the east of the country over time, and the degree of its concentration increased. On the contrary, northeastern China continued to have a lower incidence. From 2004 to 2009, the incidence of influenza in southwestern, northwestern, and northern China were consistently higher than the national average. In 2009, the na-

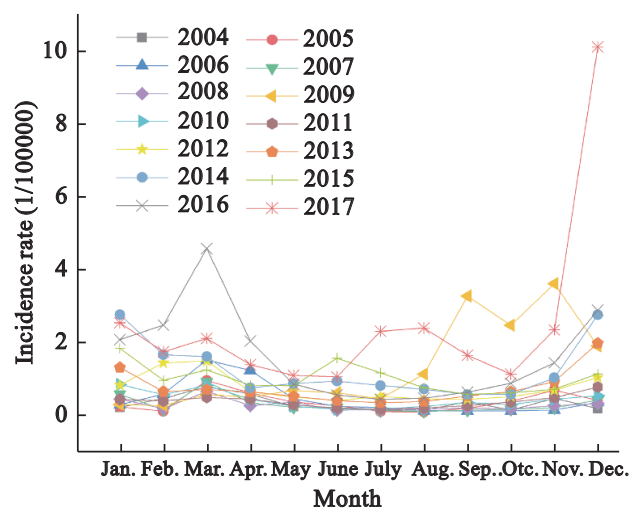

Fig. 2 Monthly incidence of influenza in China (excluding Hong Kong, Macau and Taiwan) during 2004-2017 tionwide incidence (except in the northeastern China) was the highest during 2004-2009, and the number of areas with a higher incidence than the average was the largest during this period. During 2010-2017, the number of regions, provinces, and cities with higher incidences was far greater than in the previous five years. The incidence of influenza was relatively higher in north, south, east, central, and northwest China. At the provincial level, Xinjiang, Beijing, Inner Mongolia, Hebei (Handan), Tibet, Guangxi, and Guangdong had significantly higher incidences of influenza during the study period. This might have been related to the rapid economic development and increase in the number of factories in recent years.

\subsubsection{Analysis of global spatial agglomeration}

Table 2 shows the global Moran's $I$ for the incidence of influenza and the results of the corresponding significance test for China in 2004-2017. Moran's I was higher than 0.2 and the $Z$-value was greater than the critical value of 1.96 for the normal distribution function at a significance level of 0.05 only in 2014, 2016, and 2017, i.e., it passed the significance test and was statistically significant in these years. This indicates that the incidence of influenza exhibited a certain spatial autocorrelation and agglomeration. However, the global Moran's I for the incidence of influenza from 2004 to 2013 and in 2015 did not exceed 0.2, indicating that the global spatial autocorrelation was not strong in these years and the interregional spatial difference in influenza was insignificant, showing a random distribution.

\subsubsection{Analysis of local spatial agglomeration}

Fig. 4 shows the characteristics of the local spatial autocorrelation for the incidence of influenza through the detection of hotspots for the flu. Areas with a regional agglomeration of incidence of influenza were registered in each year. From 2004 to 2009, significant hotspots with higher incidences formed in Xinjiang, Tibet, and Gansu, while areas with few cases were concentrated in Hebei, Henan, Jilin, and Shandong. From 2010 to 2017 , the hotspots gradually shifted eastward from Shaanxi, Shanxi, Beijing, and Hebei to Guangdong, Fujian, and Guangxi, while areas with low incidence were concentrated in Sichuan, Guizhou, Heilongiiang, Jilin, and Liaoning. In general, hotspots of influenza tended to shift from the northwest to the southeast, possibly owing to the limited economic development of western regions of China earlier on. In the later period, eastern re- 

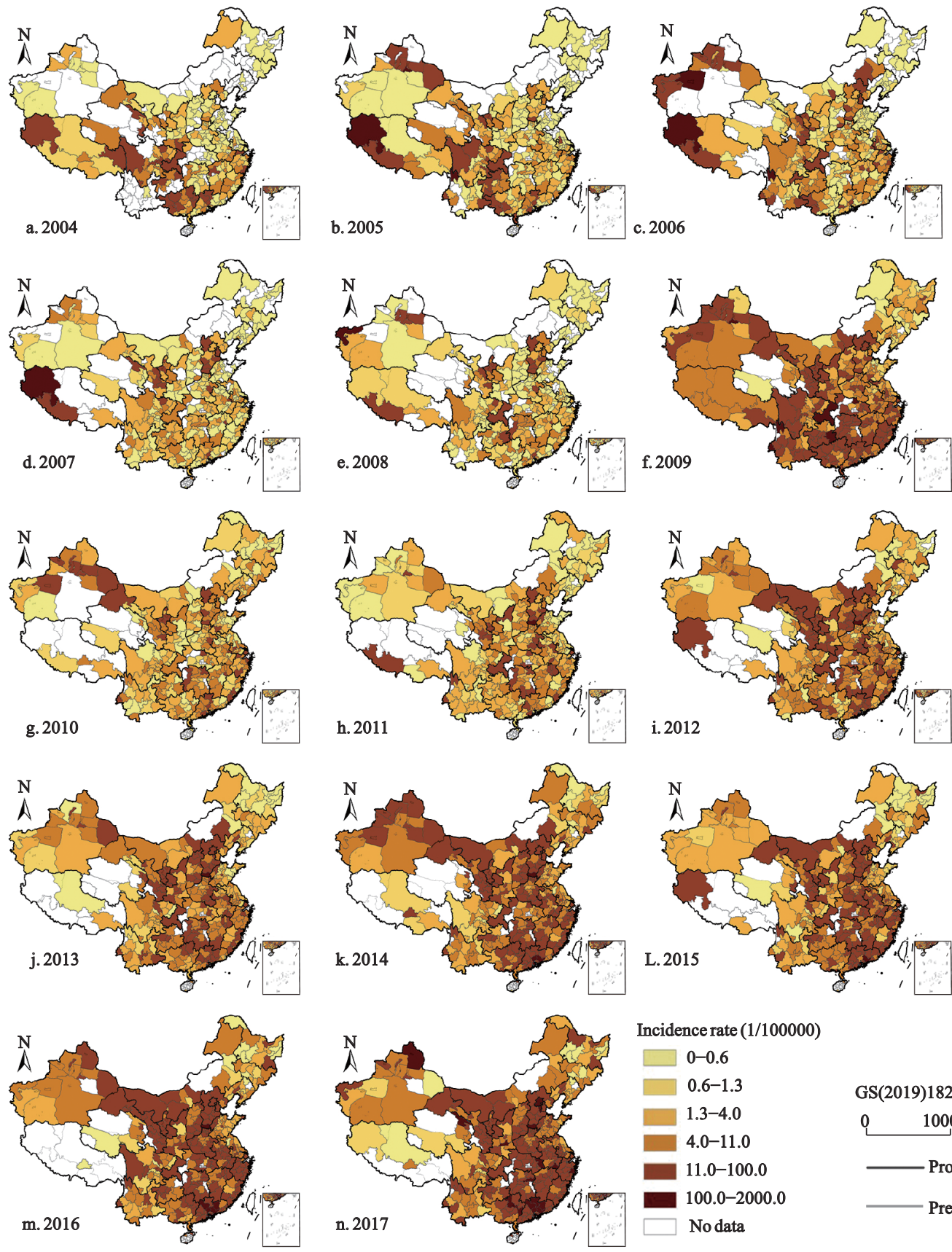

Incidence rate $(1 / 100000)$

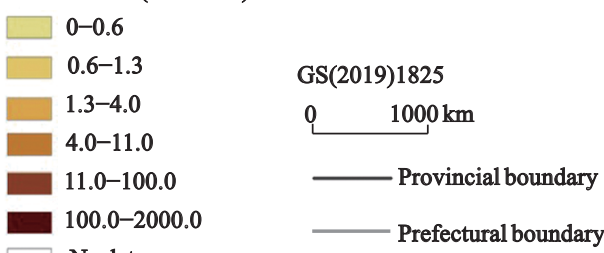

Fig. 3 Spatial distribution of yearly incidence of influenza in China during 2004-2017

Table 2 Global Moran's I analysis of influenza incidence in China (excluding Hong Kong, Macau and Taiwan) during 2004-2017

\begin{tabular}{|c|c|c|c|c|c|c|c|c|c|}
\hline Year & Moran's $I$ & $Z$ value & $P$ value & Spatial pattern & Year & Moran's $I$ & $Z$ value & $P$ value & Spatial pattern \\
\hline 2004 & 0.105 & 6.240 & 0.000 & - & 2011 & 0.047 & 4.816 & 0.000 & - \\
\hline 2005 & 0.015 & 2.343 & 0.019 & - & 2012 & 0.084 & 7.004 & 0.000 & - \\
\hline 2006 & 0.009 & 1.250 & 0.211 & - & 2013 & 0.078 & 8.039 & 0.000 & - \\
\hline 2007 & 0.019 & 2.480 & 0.013 & - & 2014 & 0.330 & 19.222 & 0.000 & Clustering \\
\hline 2008 & 0.026 & 2.042 & 0.041 & - & 2015 & 0.153 & 15.318 & 0.000 & - \\
\hline 2009 & 0.076 & 5.511 & 0.000 & - & 2016 & 0.296 & 24.136 & 0.000 & Clustering \\
\hline 2010 & 0.022 & 2.084 & 0.037 & - & 2017 & 0.305 & 22.252 & 0.000 & Clustering \\
\hline
\end{tabular}

Note: - , no significance or no clustering 

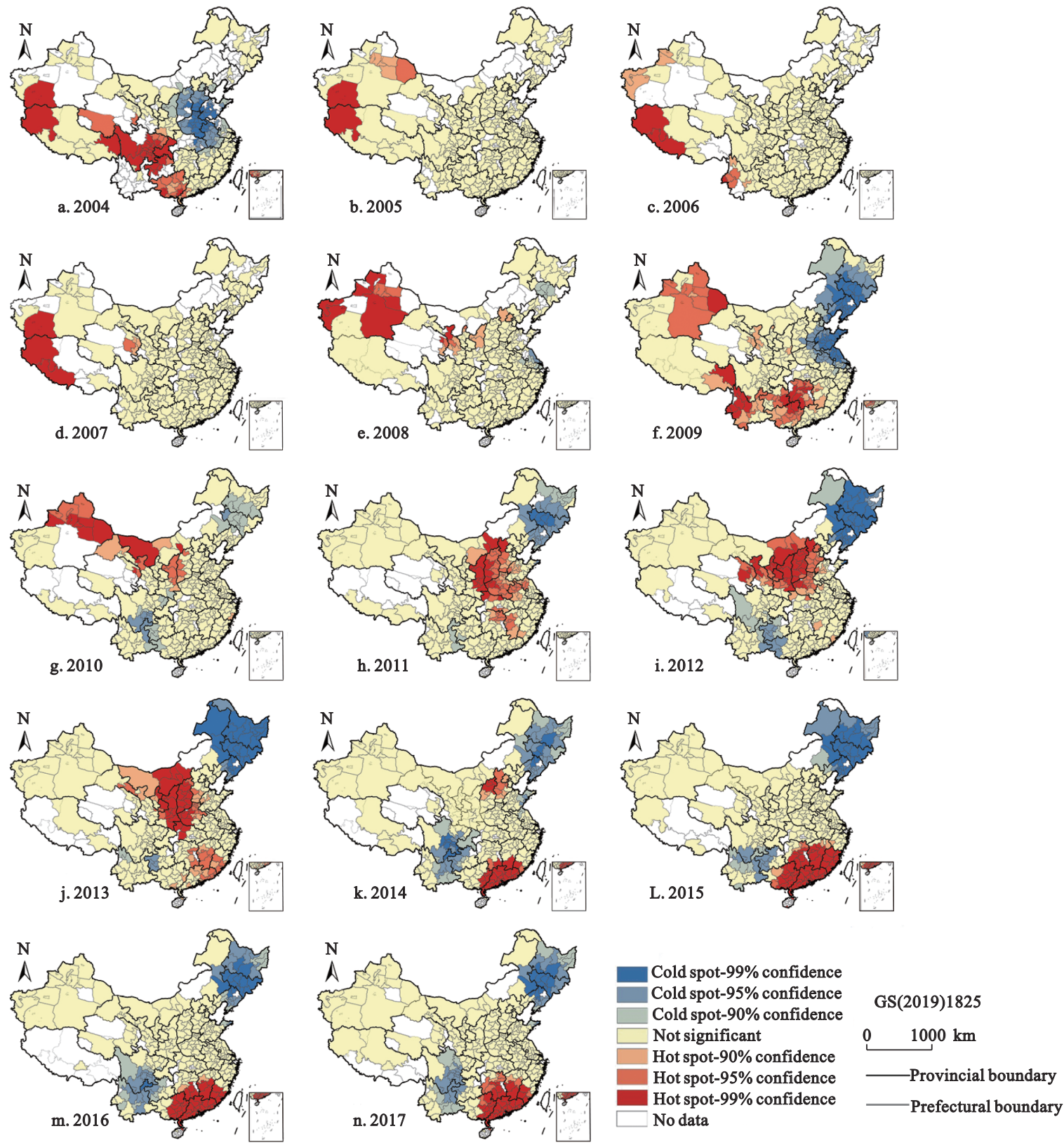

Fig. 4 Local Getis-Ord $G_{i}^{*}$ of influenza incidence in China during 2004-2017

gions might have suffered from a higher agglomeration of influenza owing to a higher population density and faster spread of pathogens, with cities such as Zhuhai, Zhongshan, and Huizhou having a higher agglomeration and incidence of influenza.

\subsection{Relationship between incidence of influenza and air pollution}

\subsubsection{Variable selection and scale determination}

Air pollution is associated with a broad spectrum of acute and chronic health effects, the nature of which may vary with the constituents of pollutants. Six air pollutants- $\mathrm{PM}_{2.5}, \mathrm{PM}_{10}, \mathrm{SO}_{2}, \mathrm{NO}_{2}, \mathrm{O}_{3}$, and $\mathrm{CO}$-were selected as factors influencing the incidence of influenza here based on the results of previous studies for which data were available. To ensure the consistency and integrity of the statistical data range, only the period from 2014 to 2017 was selected to examine these factors. Because not all results of correlation passed the ShapiroWilk test, the Spearman rank correlation analysis was used to evaluate their mutual effects as it did not have any requirement regarding the distribution of the raw data. 
The value of the correlation coefficient varied depending on the spatial scale of the research problem or the basic unit of geographical analysis used, where this is the modifiable areal unit problem (MAUP) (Robinson, 1950; Shi and Wang, 2016). Owing to the large span from the north to the south of China, the geographical conditions and related factors are significantly different, because of which the selection of different spatial scales for analysis leads to different results. Therefore, it was necessary to use a method with an independent scale to carry out statistical reasoning. To reduce the influence of the MAUP as much as possible, three spatial scales (national, provincial, and prefecture-level cities) were used to quantitatively describe the relation between influenza and air pollution.

\subsubsection{Correlation analysis at the national level}

The overall correlation coefficients for the incidence of influenza and air pollution-related factors at the national scale were calculated for 2014-2017 (Table 3). The results indicate that the rank correlation coefficients were lower than 0.3 in each year, indicating a very weak correlation. The $P$-values were mostly less than 0.01 , and led us to reject the null hypothesis that factors influencing the air quality were not related to the incidence of influenza, i.e., the occurrence of influenza was positively correlated with concentrations of $\mathrm{PM}_{2.5}, \mathrm{PM}_{10}$, $\mathrm{SO}_{2}, \mathrm{NO}_{2}$, and $\mathrm{CO}$ at the national level. However, it showed a very weak negative correlation with the concentration of $\mathrm{O}_{3}$ because the annual averages of air pollution and the incidence of influenza were too general, and specific phenomena in small areas were neglected. Therefore, correlation analyses at the national level were used only to compare differences at other spatial scales, and had no reference significance.

\subsubsection{Correlation analysis at the provincial level}

Fig. 5 shows the correlation coefficients between the incidence of influenza and air pollution for each province of China. A total of $52 \%$ of the results passed the significance test during the study period. The correlation between the incidence of influenza and air pollution was concentrated in the range from -0.5 to 0.5 in a majority of provinces in China. The values of $\mathrm{PM}_{2.5}, \mathrm{PM}_{10}, \mathrm{SO}_{2}$, $\mathrm{NO}_{2}$, and $\mathrm{CO}$ were generally higher than zero, indicating a positive correlation with influenza. The values of $\mathrm{SO}_{2}$ and $\mathrm{NO}_{2}$ indicated the highest influence. More than half of the correlation values for $\mathrm{O}_{3}$ were lower than 0 , indicating a negative correlation with the incidence of influenza. The correlations between the overall incidence of influenza and air pollution were mostly above 0.5 with a stronger correlation in Beijing, Tianjin, Shanghai, Jiangsu, Ningxia, and Shaanxi. The results for Shanghai in 2016 were the most significant, i.e., values of the correlation of influenza with $\mathrm{SO}_{2}, \mathrm{PM}_{2.5}, \mathrm{PM}_{10}$, $\mathrm{NO}_{2}$, and $\mathrm{O}_{3}$ were $0.92,0.75,0.71,0.89$, and -0.73 , respectively. Correlations between the incidence of influenza and $\mathrm{SO}_{2}$ concentrations were also significant in Beijing and Tianjin. The statistical results for Tibet, Hainan, Inner Mongolia, Chongqing, Sichuan, and Anhui did not pass the significance test. Regions with significant correlations were mainly located in the Yangtze River Delta, Beijing, Tianjin, Shaanxi, Gansu, and Ningxia. Most of them had higher incidences of influenza owing to severe air pollution.

\subsubsection{Correlation analysis at the level of prefectural level}

Fig. 6 shows the correlation coefficients between the incidence of influenza and air pollution in each prefecturelevel city in China, and the results with p-values of less

Table 3 Correlation coefficient between influenza incidence and air pollution factors in China during 2014-2017

\begin{tabular}{|c|c|c|c|c|c|c|c|c|}
\hline \multirow{2}{*}{$\begin{array}{c}\text { Air } \\
\text { pollutants }\end{array}$} & \multicolumn{2}{|l|}{2014} & \multicolumn{2}{|l|}{2015} & \multicolumn{2}{|l|}{2016} & \multicolumn{2}{|l|}{2017} \\
\hline & $\begin{array}{c}\text { Spearman's correlation } \\
\text { coefficient }\end{array}$ & $\begin{array}{c}\text { Significance } \\
(P)\end{array}$ & $\begin{array}{c}\text { Spearman's correlation } \\
\text { coefficient }\end{array}$ & $\begin{array}{c}\text { Significance } \\
(P)\end{array}$ & $\begin{array}{c}\text { Spearman's correlation } \\
\text { coefficient }\end{array}$ & $\begin{array}{c}\text { Significance } \\
(P)\end{array}$ & $\begin{array}{c}\text { Spearman's correlation } \\
\text { coefficient }\end{array}$ & $\begin{array}{c}\text { Significance } \\
(P) \\
\end{array}$ \\
\hline $\mathrm{PM}_{2.5}$ & $0.079^{* *}$ & 0.001 & 0.033 & 0.050 & $0.213^{* *}$ & 0.000 & $0.168^{* *}$ & 0.000 \\
\hline $\mathrm{PM}_{10}$ & 0.031 & 0.201 & 0.009 & 0.601 & $0.193^{* *}$ & 0.000 & $0.107^{* *}$ & 0.000 \\
\hline $\mathrm{SO}_{2}$ & $0.098^{* *}$ & 0.000 & $0.052^{* *}$ & 0.002 & $0.122^{* *}$ & 0.000 & $0.045^{* *}$ & 0.008 \\
\hline $\mathrm{CO}$ & $0.214^{* *}$ & 0.000 & $0.069^{* *}$ & 0.000 & $0.167^{* *}$ & 0.000 & $0.129^{* *}$ & 0.000 \\
\hline $\mathrm{NO}_{2}$ & $0.084^{* *}$ & 0.001 & $0.040^{*}$ & 0.019 & $0.232^{* *}$ & 0.000 & $0.175^{* *}$ & 0.000 \\
\hline $\mathrm{O}_{3}$ & $-0.240^{* *}$ & 0.000 & $-0.130^{* *}$ & 0.000 & $-0.185^{* *}$ & 0.000 & $-0.225^{* *}$ & 0.000 \\
\hline
\end{tabular}

Notes: ${ }^{*} P<0.01$ significance level; $* P<0.05$ significance level 


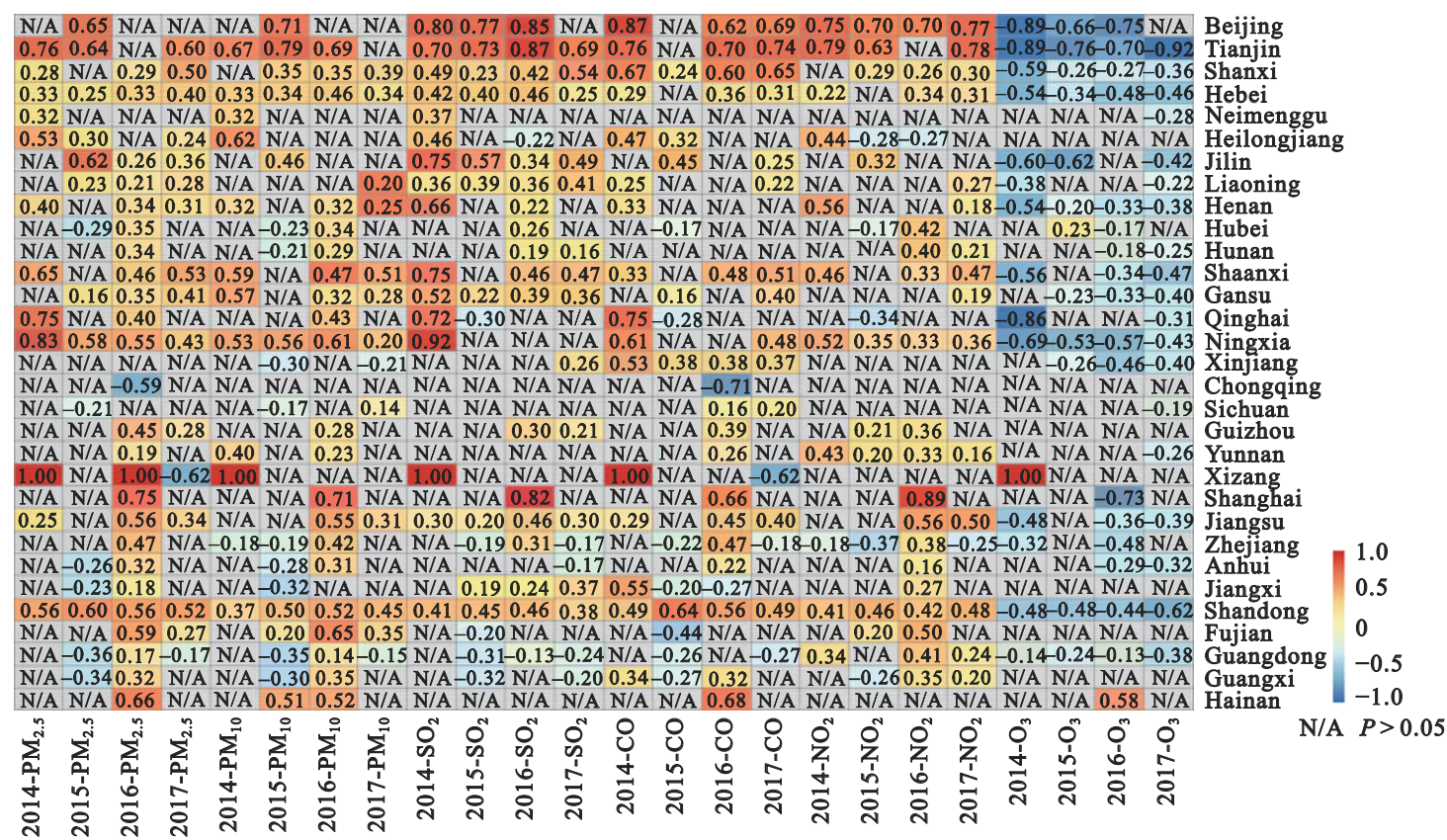

Fig. 5 Hotspots of the correlation between the incidence of influenza and air pollution of provinces, municipality and autonomous regions of China (excluding Hong Kong, Macau, and Taiwan) in 2014-2017

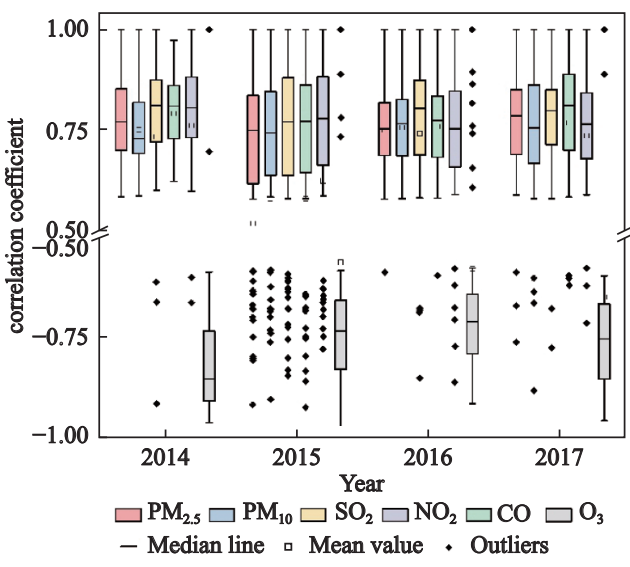

Fig. 6 Correlation chart between the incidence rate of influenza and air pollution in China's prefectural cities during 2014-2017

than 0.05 were retained. It is clear that the correlation coefficients between the concentrations of $\mathrm{PM}_{2.5}, \mathrm{PM}_{10}$, $\mathrm{SO}_{2}, \mathrm{NO}_{2}, \mathrm{CO}$, and $\mathrm{O}_{3}$, and the incidence of influenza were all above 0.5 . The first five factors had positive values, with the maximum and minimum values in the range $(0.5,1.0]$, and median values mostly concentrated around 0.75 , indicating a strong correlation. Its correlations with the concentration of $\mathrm{O}_{3}$ had negative values, with a median value concentrated around -0.750 and indicating a negative correlation. We compared the degrees of correlation between the five air pollution-related factors, which were positive correlated, with the incidence of influenza, and their absolute values showed differences that were not significant. The overall correlation between the incidence of influenza and the concentration of $\mathrm{SO}_{2}$ was the highest, and had the highest median values (mean median of 0.795 ). The average median values of $\mathrm{CO}$ and $\mathrm{NO}_{2}$ were 0.790 and 0.774 , respectively. Those of $\mathrm{PM}_{2.5}$ and $\mathrm{PM}_{10}$ were smaller, at 0.764 and 0.747 , respectively. The average median correlation coefficient between the content of $\mathrm{O}_{3}$ and the incidence of influenza was -0.763 , indicating that $\mathrm{O}_{3}$ was significantly negatively correlated with it.

\subsubsection{Summary of main control elements}

The different scales considered showed differences in the correlation values between the incidence of influenza and air pollution-related factors. At the scales of the province and prefecture-level city, the results were more statistically significant. In general, the contents of $\mathrm{PM}_{2.5}, \mathrm{PM}_{10}, \mathrm{SO}_{2}, \mathrm{NO}_{2}$, and $\mathrm{CO}$ were positively correlated with the incidence of influenza. Of them, $\mathrm{SO}_{2}, \mathrm{CO}$, and $\mathrm{NO}_{2}$ were highly correlated with influenza, followed by $\mathrm{PM}_{2.5}$ and $\mathrm{PM}_{10}$. $\mathrm{O}_{3}$ was also highly correlated with influenza, but negatively so.

\section{Discussion}

\subsection{Consideration of the data}

The data on influenza used in this study were sampled data, and influenced the results to a certain extent. We 
used data provided by Data Center of China Public Health Science, part of China Center for Disease Control and Prevention. A large number of cases of influenza recorded therein were based on clinical diagnoses, with a higher ratio of incorrect diagnosis and underreported cases (Aman A T, 2020). This is because some carriers of influenza are asymptomatic, which makes it difficult to comprehensively find, diagnose, and report cases . A study by the US Emergency Departments found that temperatures recorded in triage were lower in the morning than in the afternoon or evening. Although there is no official rate of missed diagnosis or incorrect diagnosis of influenza in China, we need to consider this factor (Harding et al., 2020). In addition, different statistical capacities in different years and regions, as well as missing data in some periods or regions due to adjustments in the administrative division, were registered. The data were not interpolated to ensure their authenticity. These problems with the data affected our method and process, and might have led to deviations between the obtained results and the empirical conditions. An analysis of the results of correlation between the incidence of influenza and air pollution revealed that the overall values that passed the significance test were not very high, where this may be related to the selection of large-scale and long-term data. However, the correlations were very significant in Beijing, Tianjin, and other places for which more basic data were likely provided. This is because large cities not only have large populations and incidence bases, but also have strong statistical and reporting-related precision, because of which the results of analysis of cities with more basic data were more accurate (Zhang et al., 2018). The availability of the results also increased successively from the national and provincial to the prefecture-levelcity scales, indicating that more explicit results were obtained at finer scales.

\subsection{Comparison with other studies}

This study focused on explaining the incidence of influenza at different spatial levels, and the results are consistent with those of cutting-edge research in epidemiology and pathology (Varon et al., 1999; Pandey et al., 2005; Tao et al., 2011; Li and Meng, 2012; Yan et al., 2013; Zhou, 2013; Li et al., 2018, 2019; Chen et al., 2018; Liu et al., 2019; Liang et al., 2019). Past work has shown that most pollution-related factors are positively correlated with the incidence of respiratory diseases, which is consistent with the results obtained in this study, i.e., air pollution has a very significant effect on cases of influenza. After entering the human body through various pathways of exposure, pollutants can interact with different target proteins, and are absorbed by the blood and human tissues. This can severely influence the defensive function of the respiratory tract, leading to an inflammatory reaction. This is an important factor in the occurrence and spread of influenza. The results of this study show that influenza is negatively correlated with the concentration of $\mathrm{O}_{3}$, and may have a specific correlation with the bactericidal characteristics of $\mathrm{O}_{3}$. However, epidemiological studies indicate that a negative correlation cannot verify the effect of interaction between the two entities considered owing to their different times of formation. The concentration of $\mathrm{O}_{3}$ tends to be higher in summer, and lower in autumn and winter, when a higher incidence of influenza has been noticed. Furthermore, long-term ozone inhalation has been attributed to increased morbidity and mortality owing to respiratory diseases, such as asthma and chronic obstructive pulmonary disease (Yang et al., 2012). In addition, the toxic effects of a number of environmental pollutants may show a lagged effect, thus posing additional challenges for scientific research on the connection between environmental pollution and health. Although scientists from different countries have provided a fair understanding of such issues as the trend, source, migration, and transformation of pollutants as well as their harmful effects on the human body, research on the characteristics of pollution, its environmental toxicology, and the risks it poses to human health remains in its infancy.

\subsection{Policy suggestion}

This study clarified the key areas for the prevention and control of influenza. The results can guide local governments in making decisions regarding medical control and environmental protection. The use of an integrated and sustainable urban planning strategy is a priority for central and local government officials. We think that the government should formulate targeted schemes for influenza prevention and control in accordance with the characteristics of different regions. The government 
should pay more attention to the Pearl River Delta, eastern China, and other areas with higher incidences of influenza, as well as cities such as Zhuhai, Zhongshan, and Huizhou. In addition, more sustainable developmental policies should be instituted and enforced to reduce the effect of air pollution on long-term economic development and the health of people. Officials should be rigorous when examining and approving new projects that involve the emissions of harmful pollutants, develop modes of pollution prevention, supervision, and accountability (Wang et al., 2017), and establish a list of enterprises that emit polluting gases such as $\mathrm{SO}_{2}, \mathrm{CO}$, and $\mathrm{NO}_{2}$. More attention should be paid to the sources of emission that increase the contents of $\mathrm{PM}_{2.5}$ and $\mathrm{PM}_{10}$. Moreover, the government should control pollution and dust in key industries, develop green transportation, optimize the industrial structure, deepen industrial pollution control, strengthen meteorological monitoring technologies and early warning management, and regulate and control emissions according to meteorological changes in a timely manner ( $\mathrm{Li}$ et al., 2020). It should ensure an environment with an appropriate concentration of $\mathrm{O}_{3}$, purified air, and engage in atmospheric disinfection and sterilization. In combination with the relevant air pollution indices, a method to calculate countrywide indices representing the risk of influenza should be formulated to provide forecasts to residents and react to outbreaks in a timely manner. A national ecological compensation plan for environmental air quality should be implemented to reduce the spread of air pollution in urban areas, and administrative divisions should be used for better prevention and control of air pollution (Sun et al., 2019).

\subsection{Research limitations and prospects}

This study has some certain limitations. First, the rising trend of the incidence of influenza observed in this study might have been related to its periodicity, or to the increased rate of reported cases of influenza and improvements in the quality of such reports. Long-term public health monitoring and epidemiological research still needs to be carried out for further verification of the trends identified here. Second, the incidence of influenza varied significantly on a daily basis in certain months in our data, because of which the monthly analysis of its incidence might have reported fewer cases certain months. The characteristics of distribution of infectious diseases at different spatial scales were different. It is thus necessary to investigate and study the disease at different scales to fully grasp the laws of its spread. We mostly used large- and medium-scale analysis, and neglected small-scale analysis. This should be carried out in future work in the area. Third, the factors influencing the incidence of influenza in China were investigated mainly from the perspective of air pollution, without considering the significant effects of natural factors such as climate and hydrology as well as social and economic factors, such as population flow and healthcare. Finally, the secondary classification of influenza was not described in detail, and epidemiological data on the age, gender, and past medical history of patients was not included. Future research should focus on improving the quality of data, examining the comprehensive mechanisms of influence of the incidence of influenza, analyze the characteristics of spatial differentiation among populations according to epidemiological principles, and conduct a comparative spatial analysis of various epidemic diseases.

\section{Conclusions}

Influenza outbreaks are a major public health problem worldwide that pose a significant threat to human life and health. This study used a top-down approach to analyze the spatio-temporal characteristics of the incidence of influenza in China, and identified and quantified the relationships between the incidence of influenza and air pollution-related parameters at different spatial scales. Three main conclusions were obtained from this study:

1) From 2004 to 2017, the incidence of influenza showed an overall trend of increase, with fluctuations and strong seasonality, in provinces, municipality and autonomous in China (excluding Hong Kong, Macao, and Taiwan). Periods of higher incidences and susceptibility occurred generally in the first and fourth quarters of each year.

2) In 2014,2016 , and 2017, the incidence of influenza in China exhibited a stronger spatial autocorrelation, and showed a pattern of agglomeration. Earlier in the study period, influenza was mainly concentrated in less developed cities and regions in northwestern China, while in recent years, it has been concentrated in densely populated cities with large population flows in 
the southeastern coastal areas, and in large and developed cities. The hotspots of influenza showed a trend of shifting from the northwest to the southeast of the country.

3) The incidence of influenza was significantly related with changes in parameters influencing air pollution. The contents of five air pollutants $-\mathrm{PM}_{2.5}, \mathrm{PM}_{10}$, $\mathrm{SO}_{2}, \mathrm{NO}_{2}$, and $\mathrm{CO}$ - were positively correlated with the incidence of influenza, and the degrees of risk posed by them from the highest to the lowest were as follows: $\mathrm{SO}_{2}>\mathrm{CO}>\mathrm{NO}_{2}>\mathrm{PM}_{2.5}>\mathrm{PM}_{10}$. The concentration of $\mathrm{O}_{3}$ showed a significant negative correlation with the incidence of influenza, but also exhibited small fluctuations due to the influence of different seasons and regions.

\section{Acknowledgments}

This work was supported by Data Center of China Public Health Science, part of China Center for Disease Control and Prevention.

\section{References}

Artois J, Jiang H, Wang X L et al., 2018. Changing Geographic Patterns and Risk Factors for Avian Influenza A(H7N9) Infections in Humans, China. Emerging Infectious Diseases, 24(1): 87-94. doi: 10.3201/eid2401.171393

Aman A T, Wibawa T, H Kosasih et al., 2020. Etiologies of severe acute respiratory infection (SARI) and misdiagnosis of influenza in Indonesia, 2013-2016. Influenza and Other Respiratory Viruses, 15(5): 34-44. doi: 10.1111/irv.12781

Bhuiyan M U, Luby S P, Alamgir N I et al., 2014. Economic burden of influenza-associated hospitalizations and outpatient visits in Bangladesh during 2010. Influenza and Other Respiratory Viruses, 8(4): 406-413. doi: 10.1111/irv.12254

Biek R, Real L A, 2010. The landscape genetics of infectious disease emergence and spread. Molecular Ecology, 19(17): 3515-3531. doi: 10.1111/j.1365-294X.2010.04679.x

Chen Jiangping, Zhang Lanlan, Yu Yuanjian et al., 2011. Analysis on epidemic of A /H1N1 Flu in mainland China based on spatial statistical methods. Geomatics and Information Science of Wuhan University, 36(11): 1363-1366. (in Chinese)

Chen R J, Yin P, Meng X et al., 2018. Associations Between Ambient Nitrogen Dioxide and Daily Cause-specific Mortality: Evidence from 272 Chinese Cities. Epidemiology , 29(4): 482-489. doi: 10.1097/EDE.0000000000000829

Dangi T, Jain B, Singh A K et al., 2014. Influenza virus genotypes circulating in and around Lucknow, Uttar Pradesh, India, during post pandemic period, August 2010-September 2012.
The Indian Journal of Medical Research, 139(3): 418-426.

Du Jian, Lan Zouran, Zhai Xinyan et al., 2018. Analysis on global outbreak situation of highly pathogenic avian influenza in 2017. China Animal Health Inspection, 35(11): 5-8. (in Chinese)

Getis A, Ord J K, 1992. The analysis of spatial association by use of distance statistics. Geographical Analysis, 24(3): 189-206. doi: 10.1111/j.1538-4632.1992.tb00261.x.

Gong Shengsheng, Xie Haichao, Chen Fahu, 2020. Spatiotemporal change of epidemics and its relationship with human living environments in China over the past 2200 years. Science China Earth Sciences, 50(5): 719-722. (in Chinese)

Guinat C, Nicolas G, Vergne T et al., 2018. Spatio-temporal patterns of highly pathogenic avian influenza virus subtype H5N8 spread, France, 2016 to 2017. European Communicable DiseaseBulletin,23(26):1700791.doi:10.2807/1560-7917.ES.2018. 23.26.1700791

Haining R P, 2003. Spatial Data Analysis: Theory and Practice. Cambridge: Cambridge University Press.

Harding C, Pompei F, Bordonaro SF et al., 2020. Fever Incidence Is Much Lower in the Morning than the Evening: Boston and US National Triage Data. Western Journal of Emergency Medicine: Integrating Emergency Care with Population Health, 21(4): 909-917. doi: 10.5811/westjem.2020.3.45215

Hayes R B, Lim C, Zhang Y L et al., 2020. $\mathrm{PM}_{2.5}$ air pollution and cause-specific cardiovascular disease mortality. International Journal of Epidemiology, 49(1): 25-35. doi: 10.1093/ ije/dyz114

Hilleman M R, 2002. Realities and enigmas of human viral influenza: pathogenesis, epidemiology and control. Vaccine, 20(25): 3068-3087. doi: 10.1016/S0264-410X(02)00254-2

Holley H L, 1998. Geography and mental health: a review. Social Psychiatry and Psychiatric Epidemiology, 33(11): 535-542. doi: 10.1007/s001270050091

Jamieson D J, Honein M A, Rasmussen S A et al., 2009. H1N1 2009 influenza virus infection during pregnancy in the USA. The Lancet, 374(9688): 451-458. doi: 10.1016/S01406736(09)61304-0

Lee S S, Wong N S, 2011. The clustering and transmission dynamics of pandemic influenza A (H1N1) 2009 cases in Hong Kong. Journal of Infection, 63(4): 274-280. doi: 10.1016/j.jinf. 2011.03.011

Lelieveld J, Evans J S, Fnais M et al., 2015. The contribution of outdoor air pollution sources to premature mortality on aglobal scale. Nature, 525(7569): 367-371. doi: 10.1038/nature15371

Li Hua, Tong Helong, Wu Xianhua et al., 2020. Spatial and temporal evolution characteristics of $\mathrm{PM}_{2.5}$ in China from 1998 to 2016. Chinese Geographical Science, 30(6): 947-958. doi: 10.1007/s11769-020-1157-5

Li Junling, Meng Ziqiang, 2012. Current progress in atmospheric environmental toxicology in China. Asian Journal of Ecotoxicology, 7(2): 133-139. (in Chinese)

Li Meifang, Ou Jinpei, Li Xia, 2016. Spatio-temporal analysis of influenza A (H1N1) in China during 2009-2013 based on GIS. 
Geographical Research, 35(11): 2139-2152. (in Chinese)

Li S, Tao H Y, Xu Y, 2013. Abiotic determinants to the spatial dynamics of dengue fever in Guangzhou. Asia-Pacific Journal of Public Health, 25(3): 239-247. doi: 10.1177/10105395 11418819

Li T T, Zhang Y, Wang J N et al., 2018. All-cause mortality risk associated with long-term exposure to ambient $\mathrm{PM}_{2.5}$ in China: a cohort study. The Lancet Public Health, 3(10): e470-e477. doi: 10.1016/S2468-2667(18)30144-0

Li Yanzhong, Zhu Hong, 2019. Time trend of incidence and mortality of notifiable infectious diseases in China from 2002-2017. Occupation and Health, 35(13): 1809-1813. (in Chinese)

Li Y S, Dong T C, Jiang X P et al., 2019. Chronic and low-level particulate matter exposure can sustainably mediate lung damage and alter CD4 T cells during acute lung injury. Molecular Immunology, 112: 51-58. doi: 10.1016/j.molimm.2019.04.033

Liang L R, Cai Y T, Barratt B et al., 2019. Associations between daily air quality and hospitalisations for acute exacerbation of chronic obstructive pulmonary disease in Beijing, 2013-17: an ecological analysis. The Lancet Planetary Health, 3(6): e270-e279. doi: 10.1016/S2542-5196(19)30085-3

Lin Jingjing, Zhang Tiewei, Li Xiuyang, 2020. Research progress on spatiotemporal clustering of disease. Chinese Journal of Epidemiology, 41(7): 1165-1170. (in Chinese)

Liu C, Chen R J, Sera F et al., 2019. Ambient Particulate Air Pollution and Daily Mortality in 652 Cities. New England Journal of Medicine, 381(8): 705-715. doi: 10.1056/NEJMoa1817364

Liu Yajie, 1985. Preliminary study on the concentration in epidemiological analysis: Brief introduction of calculation method of concentration degree. Chinese Journal of Public Health, 4(3): 43-45. (in Chinese)

Lonati G, Crippa M, Gianelle V et al., 2011. Daily patterns of the multi-modal structure of the particle number size distribution in Milan, Italy. Atmospheric Environment, 45(14): 2434-2442. doi: 10.1016/j.atmosenv.2011.02.003

Lu Bulai, Wang Jinfeng, Zeng Guang, 2010. Approaches to study disease clustering in space. Disease Surveillance, 25(5): 394-396, 409. (in Chinese)

Ma Guifeng, Zhu Jie, Cao Huijun et al., 2019. Epidemiological analysis of influenza virus in China from 2013 to 2018. Journal of Pathogen Biology, 14(1): 73-77. (in Chinese)

Mahabir R S, Severson D W, Chadee D D, 2012. Impact of road networks on the distribution of dengue fever cases in Trinidad, West Indies. ActaTropica, 123(3): 178-183. doi: 10.1016/j. actatropica.2012.05.001

Matus K, Nam K-M, Selin N E et al., 2012. Health damages from air pollution in China. Global Environmental Change, 22(1): 55-66. doi: 10.1016/j.gloenvcha.2011.08.006

Murari V, Singh N, Ranjan R et al., 2020. Source apportionment and health risk assessment of airborne particulates over central Indo-Gangetic Plain. Chemosphere, 257: 127145. doi: 10.1016/ j.chemosphere.2020.127145

National Health Commission of the people's Republic of China.
Influenza diagnosis and treatment program (2020 Edition). http://www.gov.cn/zhengce/zhengceku/2020-11/05/content_5557 639.htm, 2020-10-27. (in Chinese)

Pandey J S, Kumar R, Devotta S, 2005. Health risks of $\mathrm{NO}_{2}$, $\mathrm{SPM}$ and $\mathrm{SO}_{2}$ in Delhi (India). Atmospheric Environment, 39(36): 6868. doi: 10.1016/j.atmosenv.2005.08.004

Putri W C W S, Muscatello D J, Stockwell M S et al., 2018. Economic burden of seasonal influenza in the United States. Vaccine, 36(27): 3960-3966. doi: 10.1016/j.vaccine.2018.05.057

Qi Lanlan, Zhou Suhong, 2018. The Influence of Neighborhood Built Environments on the Spatial-temporal Characteristics of Residents' Daily Leisure Activities: A Case Study of Guangzhou. Scientia Geographica Sinica, 38(1): 31-40. (in Chinese). doi: 10.13249/j.cnki.sgs.2018.01.004

Robinson W S, 1950. Ecological correlations and the behavior of individuals. American Sociological Review, 15(3): 351-357. doi: $10.2307 / 2087176$

Rosenberg M, 2017. Health geography III: Old ideas, new ideas or new determinisms? Progress In Human Geography, 41(6): 832-842. doi: 10.1177/0309132516670054

Shi Xun, Wang Fahui, 2016. Applications of geospatial information technologies in public health. Beijing: Higher Education Press: 17-18. (in Chinese)

Shkurinskii B V, Chibilev A A, 2012. Medico-geographical assessment of socioeconomic conditions in the West Kazakhstan province. Geography and Natural Resources, 33(3): 252-257. doi: 10.1134/S1875372812030122

Skog L, Linde A, Palmgren H et al., 2014. Spatiotemporal characteristics of pandemic influenza. BMC Infectious Diseases, 14(1): 378-391. doi: 10.1186/1471-2334-14-378

Spearman C, 1904. The proof andmeasurementof association between two things. The American Journal of Psychology, 15(1): 72-101. doi: 10.2307/1412159

Sun Yanlan, Li Wei, Wu Jing et al., 2018. Correlation between meteorological factors and influenza-like illness in Xining City. Preventive Medicine Tribune, 8(5): 378-383. (in Chinese). doi: 10.16505/j.2095-0136.2018.0062

Sun Zhe, Zhan Dongsheng, Jin Fengjun, 2019. Spatio-temporal Characteristics and Geographical Determinants of Air Quality in Cities at the Prefecture Level and Above in China. Chinese Geographical Science, 20(2): 316-324. doi: 10.1007/s11769019-1031-5

Tao Y B, Zhong L J, Huang X L et al., 2011. Acute mortality effects of carbon monoxide in the Pearl River Delta of China. Science of the Total Environment, 410-411: 34-40. doi: 10.1016/j.scitotenv.2011.09.004

The Central People's Government of the People's Republic of China (CPG of PRC). Opinions of the State Council on the Implementation of Healthy China Action. http://www.gov.cn/ zhengce/content/2019-07/15/content_5409492.htm,2019-07-15. (in Chinese)

To T, Zhu J Q, Villeneuve P J et al., 2015. Chronic disease prevalence in women and air pollution - A 30-year longitudinal cohort study. Environment International, 80: 26-32. doi: 
10.1016/j.envint.2015.03.017

Trujillo M D, Plough A, 2016. Building a culture of health: A new framework and measures for health and health care in America. Social Science \& Medicine, 165: 206-213. doi: 10.1016/j.socscimed.2016.06.043

Utell M J, Frampton M W, 2000. Acute health effects of ambient air pollution: the ultrafine particle hypothesis. Journal of Aerosol Medicine, 13(4): 355-359. doi: 10.1089/jam.2000.13.355

Varon J, Marik P E, Fromm R E Jr et al., 1999. Carbon monoxide poisoning: a review for clinicians. Journal of Emergency Medicine, 17(1): 87-93. doi: 10.1016/S0736-4679(98)00128-0

Vazquez-Prokopec G M, Kitron U, Montgomery B et al., 2010. Quantifying the spatial dimension of dengue virus epidemic spread within a tropical urban environment. PLoS Neglected Tropical Diseases, 4(12): e920. doi: 10.1371/journal.pntd. 0000920

Vittecoq M, Roche B, Cohen J M et al., 2015. Does the weather play a role in the spread of pandemic influenza? A study of H1N1pdm09 infections in France during 2009-2010. Epidemiol Infect, 143(16): 3384-3393. doi: 10.1017/S09502 68815000941

Wang Jiaoe, Du Delin, Wei Ye et al., 2020. The development of COVID-19 in China: Spatial diffusionand geographical pattern. Geographical Research, 39(7): 1450-1462. (in Chinese)

Wang Jinfeng, Ge Yong, Li Lianfa et al., 2014. Spatiotemporal data analysis in Geography. Acta Geographica Sinica, 69(9): 1326-1345. (in Chinese)

Wang X, Jiang H, Wu P et al., 2017. Epidemiology of avian influenza A H7N9 virus in human beings across five epidemics in mainland China, 2013-17: an epidemiological study of laboratory-confirmed case series. Lancet Infectious Diseases, 17(8): 822. doi: 10.1016/S1473-3099(17)30323-7

Wang Zhenbo, Liang Longwu, Lin Xiongbin et al., 2017. Control Models and Effect Evaluation of Air Pollution in Jing-JinJi Urban Agglomeration. Environmental Science, 38(10): 4005-4014. (in Chinese). doi: 10.13227/j.hjkx.201701007

Wen T H, Lin M H, Teng H J et al., 2015. Incorporating the human-aedes mosquito interactions into measuring the spatial risk of urban dengue fever. Applied Geography, 62: 256-266. doi: 10.1016/j.apgeog.2015.05.003

World Health Organization. Influenza (seasonal). Available at http://www.https://www.who.int/home. 2018-01-02.

World Health Organization (WHO). Influenza Update $N^{\circ} 305$.
Available at https://www.who.int/influenza/surveillance monitoring/updates/2017_12_25_surveillance_update_305.pdf?ua= $1.2017-12-25$.

Wu P C, Lay J G, Guo H R et al., 2009. Higher temperature and urbanization affect the spatial patterns of dengue fever transmission in subtropical Taiwan. Science of the Total Environment, 407(7): 2224-2233. doi: 10.1016/j.scitotenv.2008.11.034

Yan M L, Liu Z R, Liu X T et al., 2013. Meta-analysis of the Chinese studies of the association between ambient ozone and mortality. Chemosphere, 93(6): 899-905. doi: 10.1016/j. chemosphere.2013.05.040

Yang C X, Yang H B, Guo S et al., 2012. Alternative ozone metrics and daily mortality in Suzhou: The China air pollution and health effects study (CAPES). Science of The Total Environment, 426: 83-89. doi: 10.1016/j.scitotenv.2012.03.036

Yang Haixiang, Xiao Hong, TianHuaiyu et al., 2011. Spatial and temporal analysis of influenza A (H1N1) in Changsha city based on GIS. Occupation and Health, 27(4): 376-380. (in Chinese)

Yang Jie, Tao Yinhua, Chai Yanwei, 2019. Neighborhood Built Environment, Community Cohesion and Public Health: the Moderating Effect of Transport - Related Physical Activity. Urban Development Studies, 26(9): 17-25. (in Chinese)

Yang Linsheng, Li Hairong, Li Yonghua et al., 2010. Progress of medical geography and environmental health studies. Progress in Geography, 29(1): 31-44. (in Chinese)

Yang Zhen, Ding Qiyan, Wang Nian et al., 2018. Distribution characteristics of health vulnerability and its influence factors in China. Scientia Geographica Sinica, 38(1): 135-142. (in Chinese)

Zhang Guoqing, 2013. Analysis of epidemic and trend of notifiable infectious diseases in Shouguang city from 1990 to 2012. Jinan: Shandong University. (in Chinese)

Zhang Ping, Zhang Bai, Peter M A, 2007. Modelling Framework on Urbanization's Effect on the Infectious Disease Transmission: A Case Study on Southampton of UK. Acta Geographic Sinica, 62(2): 157-170. (in Chinese)

Zhang Xiaoxi, Zong Lian, Wang Haiyin et al., 2018. Construction of a performance evaluation framework for health systems of mega-cities. Chinese Health Resources, 21(2): 167-175. (in Chinese)

Zhou Ju, 2013. Health Effects of Air Pollution. Bulletin of Chinese Academy of Sciences, 28(03): 371-377. (in Chinese) 